\title{
Pré-cálculo na licenciatura em matemática: Revisão sistemática das contribuições pedagógicas ao desempenho do estudante
}

\author{
Pre-calculus in the mathematics degree: Systematic review of pedagogical contributions to student \\ performance
}

Precálculo en la carrera de matemáticas: Revisión sistemática de las contribuciones pedagógicas al desempeño de los estudiantes

Recebido: 26/04/2021 | Revisado: 05/05/2021 | Aceito: 07/05/2021 | Publicado: 21/05/2021

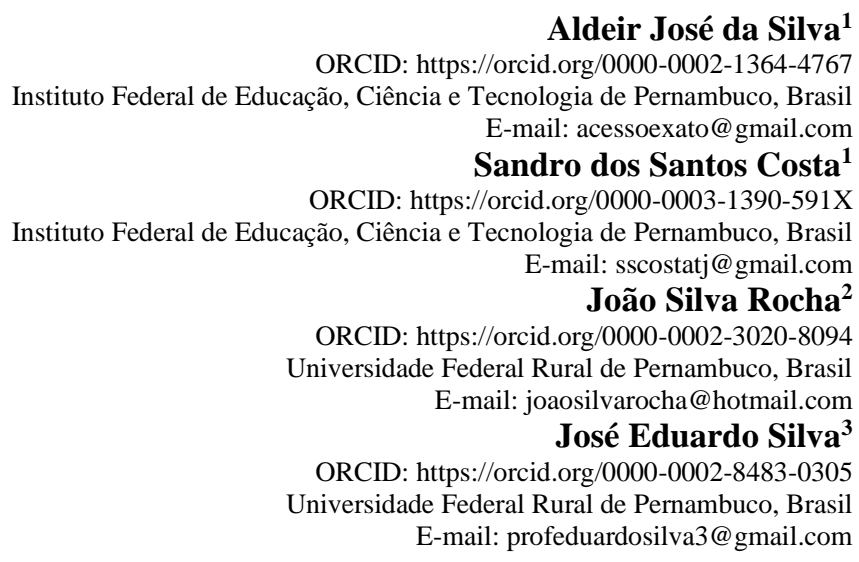

\begin{abstract}
Resumo
Os estudos sobre o componente curricular Pré-Cálculo oferecido nos anos iniciais da graduação, têm se apresentado bastante discutido no meio acadêmico, tendo em vista que o desenvolvimento do componente envolve conteúdos que requerem uma base conceitual de funções dos tipos logarítmicas e exponenciais constantes no conteúdo de matemática aplicado nos anos finais da educação básica. Objetivou-se refletir analiticamente o componente curricular Pré-Cálculo em suas abordagens conceituais no Curso de Licenciatura em Matemática, relacionadas prática didática no processo de ensino aprendizagem. Utilizou-se como metodologia, a pesquisa de natureza qualitativa e quantitativa, de caráter descritiva, exploratória e analítica. Para coleta de dados, realizou-se um levantamento bibliográfico, pelo critério de publicações atualizadas, por meio das plataformas Scielo e Google Acadêmico, considerando o período de publicações nacionais e internacionais entre 2017 e 2021. Os resultados demonstraram a dificuldade de aprendizagem na graduação que colabora para evasões e reprovações no início do curso e indicaram práticas didáticas que merecem atenção em melhorias que contribuam para uma base conceitual nos anos finais da educação básica. Pode-se concluir que as dificuldades de aprendizagem do componente nos anos iniciais da graduação, indicam a necessidade de repensar os métodos e práticas didáticas dos conteúdos matemáticos desde o ensino médio.
\end{abstract}

Palavras-chave: Pré-cálculo; Aprendizagem matemática; Educação básica.

\begin{abstract}
The studies on the Pre-Calculus curricular component offered in the initial years of graduation, have been widely discussed in the academic environment, considering that the development of the component involves contents that require a conceptual basis of functions of logarithmic and exponential types contained in the content of mathematics applied in the final years of basic education. The objective was to reflect analytically the Pre-Calculus curricular component in its conceptual approaches in the Mathematics Degree Course, related to didactic practice in the teaching-learning process. It was used as methodology, the research of qualitative and quantitative nature, of descriptive, exploratory and analytical character. For data collection, a bibliographic survey was carried out, based on the criterion of updated publications, through the Scielo and Google Scholar platforms, considering the period of national and international publications between 2017 and 2021. The results demonstrated the learning difficulty in
\end{abstract}

${ }^{1}$ Graduando em Licenciatura em Matemática pelo Instituto Federal de Educação, Ciência e Tecnologia de Pernambuco - IFPE
${ }^{2}$ Professor e Coorientador pelo Instituto Federal de Educação, Ciência e Tecnologia de Pernambuco - IFPE
${ }^{3}$ Professor e Orientador pelo Instituto Federal de Educaçâo, Ciência e Tecnologia de Pernambuco - IFPE - e-mail:silva.j.e@ outlook.com 
undergraduate courses that collaborates with dropouts and failures at the beginning of the course and indicated didactic practices that deserve attention in improvements that contribute to a conceptual basis in the final years of basic education. It can be concluded that the learning difficulties of the component in the initial years of graduation, indicate the need to rethink the didactic methods and practices of mathematical content since high school.

Keywords: Precalculus; Mathematical learning; Basic education.

\section{Resumen}

Los estudios sobre el componente curricular de Pre-Cálculo ofertados en los años iniciales de egreso, han sido ampliamente discutidos en el ámbito académico, considerando que el desarrollo del componente involucra contenidos que requieren una base conceptual de funciones de tipo logarítmico y exponencial contenidas en el contenido de las matemáticas aplicadas en los últimos años de la educación básica. El objetivo fue reflejar analíticamente el componente curricular de Pre-Cálculo en sus enfoques conceptuales en la Licenciatura en Matemáticas, relacionado con la práctica didáctica en el proceso de enseñanza-aprendizaje. Se utilizó como metodología la investigación de carácter cualitativo y cuantitativo, de carácter descriptivo, exploratorio y analítico. Para la recolección de datos se realizó un relevamiento bibliográfico, según el criterio de publicaciones actualizadas, a través de las plataformas Scielo y Google Scholar, considerando el período de publicaciones nacionales e internacionales entre 2017 y 2021. Los resultados evidenciaron la dificultad de aprendizaje en los cursos de pregrado que colabora con deserciones y reprobaciones al inicio del curso e indicó prácticas didácticas que merecen atención en mejoras que contribuyan a una base conceptual en los últimos años de educación básica. Se puede concluir que las dificultades de aprendizaje del componente en los primeros años de egreso, indican la necesidad de repensar los métodos y prácticas didácticas de los contenidos matemáticos desde el bachillerato.

Palabras clave: Precálculo; Aprendizaje matemático; Educación básica.

\section{Introdução}

No decorrer dos Cursos de Licenciatura em Matemática são desenvolvidas diversas disciplinas que envolvem cálculos complexos, e podem caracterizar como obstáculos aos estudantes. Disciplinas como a de Cálculo Diferencial e Integral exigem do estudante a compreensão de conceitos e definições necessários ao seu desenvolvimento matemático, a falta desta compreensão acarreta índices de reprovações nos anos iniciais do curso (Thomé, Duro, \& Andrade, 2020).

Torres e Soares (2017) citam que os conceitos matemáticos são construídos desde o ensino fundamental e médio, uma vez não se estabelecendo na vida do estudante, podem gerar dificuldades em seu desempenho no ensino superior, pois, a disciplina Cálculo Diferencial e Integral necessita desta base conceitual à medida que novos conceitos são atribuídos aos graduandos. Desta forma, a disciplina compõe-se de uma variedade de conteúdos algébricos e aritméticos, além de ser enraizada no estudo das funções, principalmente as logarítmicas, trigonométricas e exponenciais naturais. Contudo, os conteúdos são ensinados de uma única vez no ensino médio (Andrade, Esquincalha \& de Oliveira, 2019).

O Cálculo Diferencial e Integral, há alguns anos, tem sido tema de diversas pesquisas devido a sua relação com o enorme índice de reprovação e evasão de estudantes nos anos iniciais dos cursos relacionados com as ciências exatas, especialmente os de Matemática, nas principais universidades do Brasil (Gomes, 2018). Observa-se que este cenário não ocorre somente ao nível universitário, tendo em vista que problemas correlacionados com a aprendizagem de conceitos matemáticos estão presentes em todos os níveis de ensino (dos Santos, Henning, Taschetto, \& Girardi, 2020).

De acordo com Saccaro, França \& Jacinto (2019), a evasão é um fenômeno complexo influenciado por inúmeras variáveis, presentes em todos os níveis de ensino. Particularmente, no ensino superior à evasão é entendida como o abandono do estudante, representando para as Instituições desperdício de recursos financeiros. Todavia, estudos relacionados aos índices que revelam este fenômeno são importantes para às Instituições Educacionais, assim como conhecer sua causa e buscar soluções e alternativas que resultem na diminuição das reprovações e desistências (Diefenthäler, 2017).

Neste contexto a disciplina de Pré-Cálculo caracteriza uma fase mediadora à disciplina de Cálculo Diferencial e Integral, apesar de que não são abordados todos os assuntos da matemática elementar. Posto isso, geralmente o tema predominante nesta disciplina, recai sobre o estudo das funções, por ser um eixo estruturante da matemática. Cabe dar ênfase a 
necessidade do comprometimento e força de vontade com os estudos para que se obter êxito nas disciplinas (de Medeiros Rosa, Alvarenga \& dos Santos, 2019).

Sendo, portanto, a disciplina Pré-Cálculo oferecida em diversas instituições educacionais, logo o conhecimento de seus principais eixos temáticos e didáticas de ensino e aprendizagem são indissociáveis ao seu desenvolvimento nos anos iniciais da graduação (Bianchini, Lima \& Gomes, 2019). Na busca por respostas para os baixos desempenhos de alguns estudantes em Cálculo Diferencial e Integral, e tais dificuldades em matemática, são traços da história social de cada estudante, geram receios, medos, problemas ao expressar-se e baixa autoestima, que impedem certas transformações com o saber.

Lopes e Fürkotter (2020), citam o aspecto da formação do professor como um processo fundamental para o embasamento teórico e prático na sua formação docente, tal aspecto se materializa desde uma concepção pedagógica ampla e objetiva que se inicia com as políticas públicas de cunho regulatório, normatizador e orientativa. Convergindo para o amparo legal das instituições e seus atores, assim o instrumento didático Projeto Político de Curso principia o norteamento de ações pedagógicas.

Desse modo, o objetivo desta pesquisa é refletir de forma analítica o componente curricular Pré-Cálculo em suas abordagens no Curso de Licenciatura em Matemática, relacionadas à prática pedagógica e a aprendizagem. Firmados neste objetivo, busca-se responder: de que forma a disciplina de Pré-Cálculo, pode contribuir para o desenvolvimento de conhecimentos significativos aos estudantes de graduação de Licenciatura em Matemática?

Utilizou-se o método de pesquisa qualitativa e quantitativa quanto à sua natureza, caracterizado por um levantamento bibliográfico e análise sistemática, considerando uma revisão comparativa dos últimos cinco anos, como critério de publicações atualizadas. Para constituir o material de estudo procedeu-se a busca de artigos, documentos, livros e e-books utilizando-se das plataformas Scielo e Google Acadêmico, motivo pelo qual estas plataformas oferecem praticidade e operacionalidade. Sobretudo, quando do recorte temporal que auxilia na busca por publicações recentes em períodos bem definidos.

\section{Metodologia}

A metodologia foi constituída como qualitativa e quantitativa quanto à sua natureza, cujos objetivos são de caráter descritivo, analítico e exploratório (Mazo, Oliveira \& Tomazzoni, 2021), e o delineamento refere-se à busca em fontes de papel, ou seja, produção científica publicada. Desta forma institui-se pesquisa de revisão bibliográfica com análise sistemática, baseada em documentos e textos diversos (Menezes, Duarte, Carvalho \& Souza, 2019). Deste modo, a coleta de dados foi realizada por meio das plataformas Scielo e Google Acadêmico, considerando o período de publicações (inter)nacionais, em periódicos entre 2017 e 2021.

As plataformas supraditas oferecem interface de fácil operacionalidade e desempenho pelos critérios de busca, pois a definição do recorte temporal constitui em um artifício condicionante na recuperação de conteúdos recentes e atualizados. Com ênfase aos eixos temáticos, ensino de matemática, pré-cálculo, licenciatura em matemática e formação do professor. Foram recuperados 118 (cento e dezoito) artigos dentre periódicos e eventos como anais de congresso e simpósio, 5 (cinco) documentos e 8 (oito) e-books.

Após a leitura dos respectivos resumos, caputs e apresentações, foram selecionados 31 (trinta e um) artigos, 3 (três) $e$ books e um documento, com relação direta ao temário desta pesquisa. Que desta forma entende-se que a pesquisa se constitui na classificação do método exploratório e descritivo (Pereira et al., 2018).

A análise dos dados inclinou-se por uma argumentação comparativa e qualitativa em função dos conteúdos temáticos, cuja organização e categorização dos dados garimpados estão vinculados aos respectivos eixos temáticos (Bardin, 2011). Posto isso, esta análise procedeu-se com a seleção de artigos que versam em seus títulos a licenciatura, ensino de matemática, pré- 
cálculo, professor de matemática e formação do professor, com a utilização de um recurso disponível on-line, por meio do site WordArt.com, conhecido por nuvem de palavras, em que as palavras com maior frequência nos títulos dos artigos se destacam na nuvem.

\section{Didática no Ensino do Pré-Cálculo}

O Sistema Educacional Brasileiro sofre com baixos índices educacionais, especialmente ao que se refere às ciências exatas, em sua maioria, os problemas começam nas primeiras aulas dos anos iniciais do Ensino Fundamental e tornam-se mais explícitos nos primeiros meses das graduações, pois neste período surgem as frustrações de estudantes diante de suas carências de conhecimentos dos conteúdos prévios ao contexto dos cursos superiores, cujos componentes curriculares e respectivos conteúdos apresentam elevado grau de dificuldades quanto ao aprendizado (Torres \& Soares, 2017).

Em meio a este contexto, nem todos os estudantes conseguem se encaixar nas propostas acadêmicas da graduação, por não conseguirem encontrar sentido naquele cenário acadêmico, que também está interligado a determinados contextos sociais. De acordo com De Moraes, Azevedo, Da Silva Vieira e Abar (2019) para que um estudante obtenha sucesso na vida acadêmica, é imprescindível que esteja bem consigo mesmo, que haja sempre equilíbrio emocional. Ou seja, para que o aprendizado e o conhecimento sejam despertos, significando a necessidade para que o estudante compreenda o que está ocorrendo ao seu redor capaz de fazer as próprias conexões produzindo sentido à sua vida e à realidade. As dificuldades de aprendizagem requerem análise de contextos múltiplos, onde aspectos sociais, econômicos e culturais são indissociáveis na apresentação de dados educacionais.

Por conseguinte, a disciplina de Pré-Cálculo tem como uma de suas principais propostas, proporcionar uma base sólida da matemática elementar aos universitários, principalmente aos ingressos dos cursos de Matemática. Isto acontece nos períodos iniciais da graduação, representando um dos primeiros contatos do estudante com a universidade (Andrade, Esquincalha \& de Oliveira, 2019). Portanto, é de interesse deste componente curricular familiarizar os estudantes aos demais componentes curriculares introdutórios da graduação em matemática, em especial, Cálculo Diferencial e Integral (de Andrade \& da Conceição Esquincalha, 2020a).

Compreendendo que para um problema ser solucionado torna-se imprescindível que se entenda suas causas, é de conhecimento da comunidade acadêmica que uma das principais dificuldades que estes estudantes enfrentam está relacionada à passagem do ensino médio para o superior nos mais diversos aspectos, como as caraterísticas acadêmica, os novos conceitos relativos aos estudos, os conteúdos das disciplinas em conjunto com a velocidade aos quais os mesmos são abordados entre outros (dos Santos Rocha, Santarosa \& Spohr, 2018). Não obstante, considerar que deve-se perceber e conhecer a realidade escolar da educação básica, dado que componentes curriculares diferem entre estados e segmento institucional, ou seja, a rede privada e a rede pública seguem orientações legais, contudo, operacionalizam os componentes em modos distintos.

Além de ser essencial na revisão de conteúdos elementares de matemática, o componente curricular Pré-Cálculo é extremamente útil para que os estudantes façam uma autorreflexão se realmente estão inseridos na área acadêmica que pretendem seguir, evitando, por exemplo, a evasão (de Moraes, Azevedo, da Silva Vieira \& Abar, 2019). Observa-se que o Pré-Cálculo tem relevância não apenas como componente curricular introdutório e preparatório, mas aponta para a utilidade na vida do estudante ao longo do curso, bem como no âmbito pessoal e considerando a variedade dos diferentes níveis acadêmicos e sociais, que muitas vezes são negligenciados pela própria dinâmica no desenvolvimento do componente.

Entender o processo de transição do ensino médio para o nível universitário fomenta compreender as práticas didáticas, considerando as competências do professor construídas ao longo de sua formação (Guérios \& Gonçalves, 2019), em seus diferentes aspectos, visando dar o suporte contemplando questões que dizem respeito às necessidades dos estudantes, à 
demanda dos professores e os conteúdos do componente curricular, possibilitando a adaptação e melhoria no desempenho dos estudantes (dos Santos Rocha, Santarosa \& Spohr, 2018).

Para isto, utiliza-se das principais ferramentas de trabalho, apesar de as ementas serem vagas, pois, a maioria dos professores usa diversas fontes, em grande parte do ensino médio. Porém, nos conteúdos algébricos, conforme expõe Dos Santos, Henning, Taschetto, e Girardi (2020) o tema predominante é o de Funções, visto que o componente curricular Cálculo Diferencial e Integral tem como base o estudo dos limites, derivadas e integrais de funções reais de uma variável real, as mais abordadas nas ementas de Pré-Cálculo são: as logarítmicas, exponenciais, trigonométricas, polinomiais e modulares com predomínio das duas primeiras. Com isto, percebe-se que à didática no Pré-cálculo visa suprir um dos principais problemas dos estudantes, que é à falta de aptidão algébrica e de resolução de problemas.

Os livros do ensino médio são os mais adotados pelos professores, dentre eles a coleção "Fundamentos de Matemática Elementar", da Editora Atual, cujos volumes mais utilizados são 1, 2, 3, 6 e 8. De forma complementar outros livros também são utilizados em algumas instituições educacionais como a coleção do Autor Luiz Roberto Dante e a coleção PROFMAT, da editora SBM (Andrade, Esquincalha \& de Oliveira, 2019). Interessante mencionar que apesar de os livros didáticos ainda serem predominantes na didática do ensino do Pré-Cálculo, nota-se que há um avanço na utilização das tecnologias digitais nesta disciplina, como a utilização de slides e do GeoGebra nas aulas (da Silva Saadi, Machado \& Pereira, 2020).

Logo, alguns universitários ao se depararem com componente curricular Pré-Cálculo conjecturam que a mesma tem apenas a função de proporcionar-lhes um embasamento teórico e prático da matemática elementar, visando um real e eficaz desempenho em componentes curriculares posteriores, como Cálculo Integral e Diferencial. Porém, ter esta conjectura é relegar à oportunidade de enriquecer significativamente os conhecimentos, sem contar ser um equívoco acreditar que o PréCálculo se relaciona tão somente com os eventuais componentes curriculares do primeiro período da Licenciatura em Matemática. Infere-se a importante consciência que os demais componentes curriculares da graduação, bem como nos níveis de mestrado, doutorado e demais ramificações da matemática, estão intimamente relacionadas aos conhecimentos matemáticos elementares (Esquincalha, de Andrade \& de Oliveira, 2020).

De acordo com De Andrade, e da Conceição Esquincalha (2020c), o componente curricular Pré-Cálculo tem início no primeiro período, mas não termina. São inúmeras as contribuições proporcionadas aos graduandos, engendrando um elo entre os conhecimentos adquiridos na universidade e à maneira pela qual os mesmos podem e devem ser compartilhados com a sociedade. Observa-se que por meio da educação é possível conquistar espaços, contribuir para o desenvolvimento e crescimento do país, bem como para a constituição de uma sociedade mais justa e igualitária diante da formação cidadã crítica e reflexiva.

\section{Aprendizagem Matemática em Pré-Cálculo}

Assim como em todas às Ciências, na Matemática existem inúmeros desafios de aprendizagem quanto aos seus conteúdos que vão desde a didática dos professores e os assuntos abordados, até o ambiente de aprendizagem, ou seja, há um verdadeiro processo de adaptação e construção (Vargas et al., 2020). Uma construção que revela desafios para a comunidade acadêmica, visto que encontrar soluções para conter as dificuldades de aprendizagem acompanhadas de reprovações e consequentes evasões. Apesar da existência de bons materiais didáticos, preparação dos professores em suas práticas pedagógicas, utilização de ferramentas tecnológicas em sala de aula, não se mostram suficientes para las superar (de Oliveira, Gonçalves \& Piasson, 2018).

Dos Santos, Henning, Taschetto e Girardi (2020) citam que há uma preocupação com os estudantes dos cursos que contém componentes curriculares ligados à Matemática, com a permanência desses estudantes nos cursos que escolheram. No que se refere ao componente curricular Cálculo Diferencial e Integral, de acordo com Torres e Soares (2017) às taxas de 
reprovação neste componente, no Instituto Federal da Paraíba - IFPB, são preocupantes. E, com a realização de uma coleta de dados com os estudantes do curso de Licenciatura em Matemática, nos períodos de 2015.2 e 2016.1, constatou-se um índice em torno de $70,8 \%$ e $91,7 \%$, respectivamente de reprovação.

Situação similar vem ocorrendo na Universidade Federal de Goiás - UFG, conforme mencionam De Medeiros Rosa, Alvarenga, \& dos Santos (2019), dos 2090 alunos matriculados no componente curricular Cálculo Diferencial e Integral - CDI, no segundo semestre de 2016, 1163 (55,65\%) foram reprovados. Considerando como situação recorrente neste nível alguns estudantes entendem como uma situação normal. Os resultados destas pesquisas não devem ser considerados como referência ao fenômeno da reprovação ou evasão, dado que são situações pontuais e não globais.

A pesquisa realizada por De Oliveira, Gonçalves e Piasson, (2018), indicam que há um enorme índice de reprovações no componente CDI nas principais Instituições Federais de Ensino Superior do Estado do Rio de Janeiro. Conforme o referido estudo, dentre outros fatores, tais dificuldades na compreensão dos conteúdos matemáticos estão relacionados a lacunas que os estudantes trazem da Educação Básica com pouca habilidade em trabalhar conceitos de frações algébricas e fatorações, por exemplo. O que não significa afirmar que esta condição se aplica direta e indiretamente as práticas pedagógicas e conteúdos desenvolvidos nas instituições de educação básica.

Posto o cenário ao qual o Pré-Cálculo está inserido, instituições de ensino superior têm concedido esforços no intuito de proporcionar melhor aprendizagem para todos os componentes curriculares. Fatores como reprovação e evasão, dentre outros, envolvem questões psicológicas pelos estudantes e a questão de organização dos pensamentos (de Jesus, \& Souto, 2018). Estes fatores são contributos no desenvolvimento da aprendizagem, portanto, considera-los em métodos de cunho intencionais é percorrer a construção de uma aprendizagem significativa.

Isto quer dizer que o interesse de toda equipe docente e gestora reside em trabalhar não apenas o lado acadêmico com os graduandos, mas também o lado emocional, as motivações que os levaram à universidade, tentando compreendê-los e proporcionando-lhes as melhores contribuições pedagógicas (de Andrade \& da Conceição Esquincalha, 2020a). Consequentemente o componente curricular Pré-Cálculo, em meio a este contexto, deve-se considerar no ingresso de estudantes um momento em que se torna necessário conhecer motivações, perfil e a predisposição destes para a profissão, à medida que é preciso dar noções sobre o que é ser professor.

A princípio, a aprendizagem no Pré-Cálculo apresenta eventuais diferenças básicas entre as universidades, de turma para turma, de professor para professor. Porém, a metodologia de ensino em várias instituições educacionais que ofertam este componente curricular objetiva reconstruir saberes relativos ao ensino da matemática elementar, oferecendo um espaço em que os licenciandos possam trabalhar com noções matemáticas fundamentais (Andrade, Esquincalha \& de Oliveira, 2019). Por questões diversas, as instituições de ensino como proponentes de metodologias neste sentido, promovem o nivelamento mínimo possível entre os estudantes, fato que não se deve entender solução definitiva na erradicação do fenômeno evasão.

Um dos fatores fundamentais na aprendizagem dos conteúdos ofertados está relacionado à resolução de muitos exercícios e uma boa didática do professor (Diefenthäler, 2017). Conjuntamente com as aplicações em momentos de aula, questões inerentes à organização da disciplina e a conduta docente são determinantes para a aprendizagem e o bom desempenho dos estudantes (de Moraes, Azevedo, da Silva Vieira \& Abar, 2019). Ressalta-se o alerta para um método de resolução de exercícios, visto que os resultados podem não constituir em aprendizagem, mas a memorização e repetição operacionais.

Nesta perspectiva observa-se que, quanto aos conteúdos há uma tendência dos professores trabalharem mais as Funções, dado que o Cálculo Diferencial e Integral é fundamentado neste eixo matemático. É imprescindível que se saiba desenvolver os conceitos de funções para se obter êxito no CDI e, consequentemente na graduação. Cabe ressaltar que na aprendizagem do Pré-Cálculo os professores se fundamentam na necessidade de proporcionar aos estudantes o saber identificar 
e diferenciar os objetivos matemáticos, ou seja, que não se prendam a fórmulas, mas que sejam capazes de deduzir as etapas dos respectivos conteúdos (Torres \& Soares, 2017).

No conteúdo função, Biazutti, Vaz \& Andrade (2020) afirmam que os professores trabalham para fazer com que os estudantes consigam entender e resolver aplicações contextualizadas, que tendem a forçar ainda mais o raciocínio dos mesmos e, além de estimulá-los a desenvolver tais informações no plano cartesiano, que saibam utilizar os gráficos. Pensando na parte mais abstrata da matemática, um tópico recorrente nas ementas do componente curricular Pré-Cálculo é a lógica matemática e teoria dos conjuntos que, além de estimular a aprendizagem, força os estudantes a argumentar, compreender e produzir pequenas demonstrações (Andrade, Esquincalha \& de Oliveira, 2019). Desde que no processo metodológico evidencie o protagonismo do estudante com autonomia.

Outro importante recurso de aprendizagem conforme Silva et al. (2019), é a utilização das inúmeras ferramentas digitais em sala de aula, estas têm se mostrados extremamente úteis na aprendizagem do Pré-Cálculo, especialmente a utilização de softwares como o GeoGebra. Este aplicativo dinâmico e gratuito combina conceitos de geometria e álgebra demonstrando essencialmente ser útil nas aulas de funções e nas exemplificações de algumas aplicações contextualizadas e aplicações gráficas. A utilização de recursos tecnológicos está relacionada com a capacidade estrutural disponível, oferecida e acessível por todos os atores envolvidos, onde os aspectos sociais não podem ser negligenciados.

No contexto em que a Sociedade Mundial, imersa nas consequências e enfrentamento da Pandemia causada pelo Covid-19, torna-se essencial para comunidade acadêmica apropriar-se dos inúmeros recursos tecnológicos para o ensino, incentivando e estimulando os estudantes a tomarem posse das novas ferramentas tecnológicas e metodologias de ensino voltadas para o ensino híbrido ou remoto (de Moraes, Azevedo, da Silva Vieira \& Abar, 2019).

Devido a sua importância, a utilização de ferramentas tecnológicas para a resolução de problemas diante da Competência Cinco da Base Nacional Comum Curricular - BNCC: compreender, utilizar e criar Tecnologias Digitais de Informação e Comunicação - TDIC, de forma crítica, significativa, reflexiva e ética nas diversas práticas sociais (incluindo as escolares) para se comunicar, acessar e disseminar informações, produzir conhecimentos, resolver problemas e exercer protagonismo e autoria na vida pessoal e coletiva (Brasil, 2017). O tempo e a determinação dos graduandos são imprescindíveis para os estudos, os quais se configuram fundamentais para a aprendizagem matemática, dadas as condições necessárias.

Destarte, pode-se dizer que o Pré-Cálculo é um componente curricular introdutório ao estudo do Cálculo Diferencial e Integral, dedicado a revisar e aprofundar conhecimentos da matemática elementar. Embora, não obstante, considerar que esse fundamento deveria apresentar-se de forma satisfatória aos conhecimentos dos estudantes recém-egressos da Educação Básica. E, por consequência de seu conjunto de seus conteúdos programáticos e objetivos, a disciplina foi integrada aos currículos visando à acomodação dos estudantes quanto ao entendimento e domínio de conhecimentos fundamentais e relevantes ao estudo do cálculo (Diefenthäler, 2017).

Por fim, o aporte de metodologias e técnicas pedagógicas depende não somente de infraestrutura ou competência do professor, mas de um reordenamento das políticas públicas norteadoras e condicionantes à efetivação do ensino e aprendizagem em todo o sistema educacional brasileiro.

\section{Resultados e Discussão}

Os eixos temáticos, Ensino de Matemática, Formação do Professor e Pré-Cálculo, subsidiaram o primeiro passo de busca por publicações científicas, (inter)nacionais, nas plataformas, Google Acadêmico e Scielo, o segundo consistiu no recorte temporal característico de uma pesquisa longitudinal que compreendeu o período dos últimos cinco anos, no sentido de recuperar publicações mais recentes e atualizadas quanto ao temário. 
O terceiro e no último passo tratou-se da importância e relevância das publicações frente aos conteúdos temáticos, sobretudo, relacionados à métrica de qualidade e influência dos periódicos em meio científico, estabelecido pela Coordenação de Aperfeiçoamento de Pessoal de Nível Superior - CAPES, que se refere ao indicador Qualis, onde os conceitos A e B entende-se como referência ao impacto, abrangência e importância relacionados à qualidade de publicações. Em síntese, os passos sobreditos constituíram o critério de coleta de dados desta pesquisa, resultando na seleção de 21 artigos científicos relacionados na Tabela 1 .

Tabela 1. Artigos em destaque recuperados das plataformas Google Acadêmico e Scielo, publicados no período [2017-2021].

\begin{tabular}{|c|c|c|c|c|}
\hline \multicolumn{5}{|c|}{ Plataforma Scielo } \\
\hline Eixo Temático & Periódico & ISSN & QUALIS/CAPES & QUANT. \\
\hline $\begin{array}{c}\text { Ensino de } \\
\text { Matemática }\end{array}$ & $\begin{array}{c}\text { Ciência e Educação } \\
(1) \text { e (2) }\end{array}$ & $1980-850 X$ & $\mathrm{~A} 2$ & 2 \\
\hline $\begin{array}{c}\text { Ensino de } \\
\text { Matemática }\end{array}$ & $\begin{array}{c}\text { Educação em Revista (Ufmg - Online) } \\
\text { (3) }\end{array}$ & $1982-6621$ & A1 & 1 \\
\hline $\begin{array}{l}\text { Ensino de } \\
\text { Matemática }\end{array}$ & Bolema : Boletim de Educação Matemática (Online) (4) & $1980-4415$ & A1 & 1 \\
\hline $\begin{array}{l}\text { Ensino de } \\
\text { Matemática }\end{array}$ & $\begin{array}{c}\text { Educar em Revista } \\
(5)\end{array}$ & $1984-0411$ & A1 & 1 \\
\hline $\begin{array}{c}\text { Ensino de } \\
\text { Matemática }\end{array}$ & $\begin{array}{l}\text { Cadernos de Pesquisa (Fundação Carlos Chagas. Online) } \\
(6)\end{array}$ & $1980-5314$ & A1 & 1 \\
\hline $\begin{array}{l}\text { Formação do } \\
\text { Professor } \\
\end{array}$ & $\begin{array}{l}\text { Bolema : Boletim de Educação Matemática (Online) } \\
(7) \text { e (8) }\end{array}$ & $1980-4415$ & A1 & 2 \\
\hline $\begin{array}{l}\text { Formação do } \\
\text { Professor }\end{array}$ & $\begin{array}{c}\text { Educação e Realidade } \\
(9) \\
\end{array}$ & $2175-6236$ & A1 & 1 \\
\hline Pré-Cálculo & $\begin{array}{l}\text { Revista Paranaense de Educação Matemática } \\
(10)\end{array}$ & $2238-5800$ & $\mathrm{~A} 3$ & 1 \\
\hline \multicolumn{5}{|c|}{ Plataforma Google Acadêmico } \\
\hline Pré-Cálculo & $\begin{array}{l}\text { Revista Boem } \\
\text { (11) e (12) }\end{array}$ & $2357-724 X$ & A4 & 2 \\
\hline Pré-Cálculo & $\begin{array}{c}\text { Revista Thema } \\
(13) \text { e (14) }\end{array}$ & $2177-2894$ & A3 & 2 \\
\hline Pré-Cálculo & $\begin{array}{c}\text { Unión (San Cristobal De La Laguna) } \\
(15)\end{array}$ & $1815-0640$ & B2 & 1 \\
\hline $\begin{array}{l}\text { Ensino de } \\
\text { Matemática }\end{array}$ & $\begin{array}{c}\text { Estudos Econômicos } \\
(16)\end{array}$ & $1980-5357$ & $\mathrm{~A} 3$ & 1 \\
\hline Pré-Cálculo & $\begin{array}{l}\text { Revista Internacional de Educação Superior } \\
(17)\end{array}$ & $2446-9424$ & $\mathrm{~A} 3$ & 1 \\
\hline $\begin{array}{l}\text { Ensino de } \\
\text { Matemática }\end{array}$ & $\begin{array}{c}\text { Research, Society And Development } \\
(18),(19) \text { e (20) }\end{array}$ & $2525-3409$ & A3 & 3 \\
\hline Pré-Cálculo & $\begin{array}{c}\text { Revista VIDYA (Santa Maria. Online) } \\
(21)\end{array}$ & $2176-4603$ & $\mathrm{~A} 3$ & 1 \\
\hline
\end{tabular}

Fonte: Autores (2021)

Observa-se na Tabela 1 que na plataforma Scielo o eixo Ensino de Matemática representou 60\% dos artigos, sucedido pelos eixos, Formação do Professor e Pré-Cálculo, com 30\% e 10\% respectivamente. Na plataforma Google Acadêmico não foi selecionado nenhum artigo referente ao eixo Formação do Professor, e corresponderam para os eixos Pré-Cálculo 63,4\%, e Ensino da Matemática 36,4\%.

Com os artigos selecionados na plataforma Scielo enumerados de 1 a 10 (Tabela 1), para o temário ensino de matemática, observou-se que as práticas pedagógicas são desenvolvidas como um processo dinâmico e o importante papel do professor em se manter constantemente atualizado para desempenhar suas funções com proposições definidas e interativas junto aos estudantes (1). O uso de tecnologias digitais são instrumentos pedagógicos e deve ser acessível aos professores e estudantes como elementos indissociáveis ao ensino e aprendizagem (2) e (3).

Com foco no desenvolvimento cognitivo, utiliza-se da técnica de tomada de consciência sucessiva como práticas pedagógicas em que o desenvolvimento de CDI refere-se à organização, análise e aritmetização do cálculo na constituição da 
aprendizagem (4). A utilização de rigor no ensino de números irracionais na educação básica e formação inicial do professor, torna uma prática fundamental no ensino e deve-se considerar o estudante ativo, comprometido e dedicado aos estudos (5) e (6).

O eixo formação de professor, têm-se as práticas pedagógicas relacionadas ao aspecto em que o ensino da matemática não se utiliza apenas como instrumento acabado, pois a caracterização da permanência na construção de conhecimentos através de cursos e capacitações deve fazer parte do ser professor (7) e (9). Portanto, o desenvolvimento do ensino requer habilidades e técnicas objetivando uma aprendizagem significativa e igualitária sem distinção de classes sociais dos estudantes ingressos nas instituições de ensino superior (8). Quanto ao eixo pré-cálculo, observou-se a prática pedagógica desenvolvida em um processo de reforço e desenvolvimento de conceitos matemáticos em torno de funções, cuja aprendizagem se concretiza na interação do estudante (10).

A seleção de artigos realizada na plataforma Google Acadêmico resultou em onze artigos relacionados aos eixos, précálculo e ensino da matemática. Tendo o eixo pré-cálculo com referências ao exercício docente com práticas pedagógicas diferenciadas, no sentido de apoiar o desenvolvimento dos estudantes com uso de tecnologias, softwares compõem os recursos metodológicos na busca por aprendizagem significativa na aquisição de conceitos epistemológicos a matemática (11) e (13). Reside no contexto acadêmico o desafio em suprir as dificuldades de estudantes universitários no desenvolvimento de CDI, devido à falta de conhecimentos básicos de matemática quando egressos do ensino médio para ingresso no ensino superior (12) e (14).

Em (15) e (17) são apontadas investigações acerca das dificuldades dos estudantes em disciplinas de cálculos, as investigações elucidam os interesses de professores e pesquisadores em desenvolver ações pedagógicas preparatórios ao componente curricular pré-cálculo, cuja prática pedagógica pauta-se em metodologia própria. Desta forma a aprendizagem do componente tem relação com a necessidade de inserção de cursos preparatórios propositivos ao nivelamento em conceitos e definições de matemática dos estudantes (21).

Diante do temário ensino de matemática observou-se aporte teórico concernente ao levantamento de dados acerca do fenômeno evasão e reprovação (16), considerando como consequências questões sócio econômica dos estudantes e formas de ingresso no ensino superior. Quanto as práticas pedagógicas na formação do professor, identificou-se que existem abordagens teóricas que evidenciam o modelo didático tecnicista (18). Também percebeu-se que o modelo tradicional de ensino na formação de professores de matemática não atendem aos desafios que ele enfrentará na sua prática (19), por último encontraram-se abordagens históricas no ensino de matemática buscando demonstrar a evolução de conceitos e práticas pedagógicas, que traz a concepção de novas técnicas alinhadas ao contexto social do estudante como ferramentas metodológicas ao aprendizado significativo (20).

Na Figura 1 aponta-se o aspecto longitudinal da pesquisa quanto aos eixos supracitados. 
Figura 1. Aspectos longitudinais dos eixos temáticos, Ensino de Matemática, Formação do Professor e Pré-Cálculo, das plataformas, Scielo e Google Acadêmico [2017-2021].
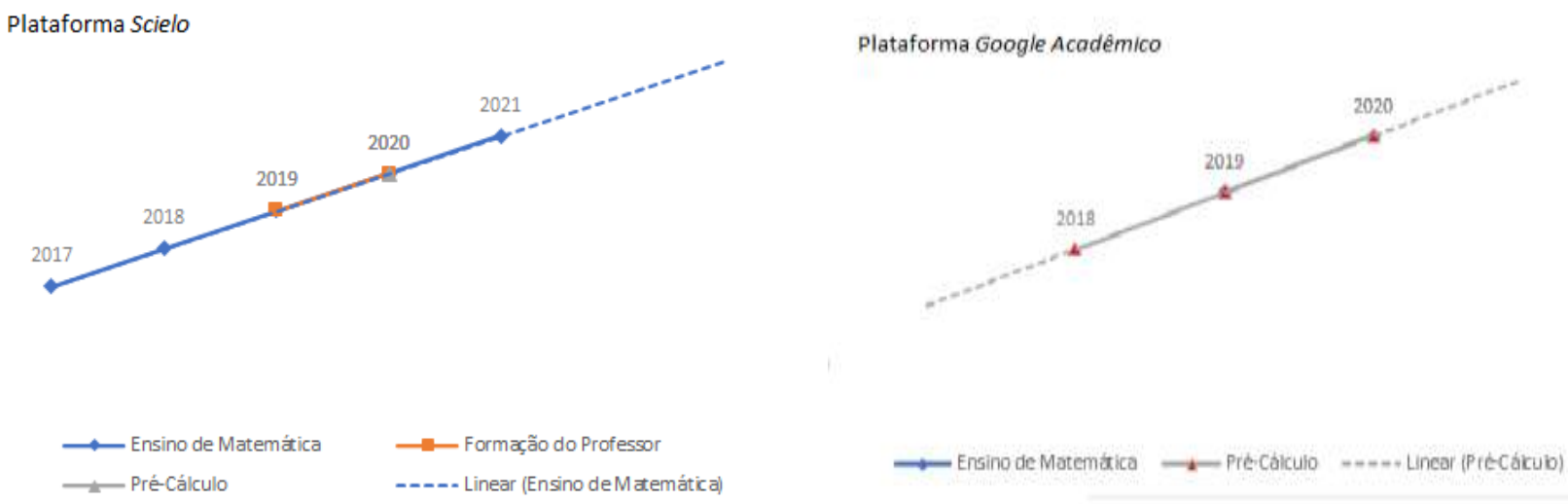

Fonte: Autores (2021)

Observa-se na Figura 1 que considerando a busca dos artigos com os critérios previamente descritos, na plataforma Scielo foi encontrado o eixo Pré-Cálculo no ano de 2020, seguido do eixo Formação do Professor nos anos de 2019 e 2020 e Ensino de matemática encontrado em todo o período, demonstrando a tendência de continuação para os próximos anos. Contudo, na plataforma Google Acadêmico o eixo Ensino de Matemática só foi verificado nos anos de 2019 e 2020 e o eixo Pré-Cálculo a partir de 2018 segue a tendência de publicação para o ano letivo. Na Figura 2 constam as palavras-chave que se destacam por sua recorrência de modo relativo nos artigos selecionados.

Figura 2. Nuvem de palavras originadas a partir dos títulos dos artigos pesquisados, depositados na plataforma Scielo e Google Acadêmico.

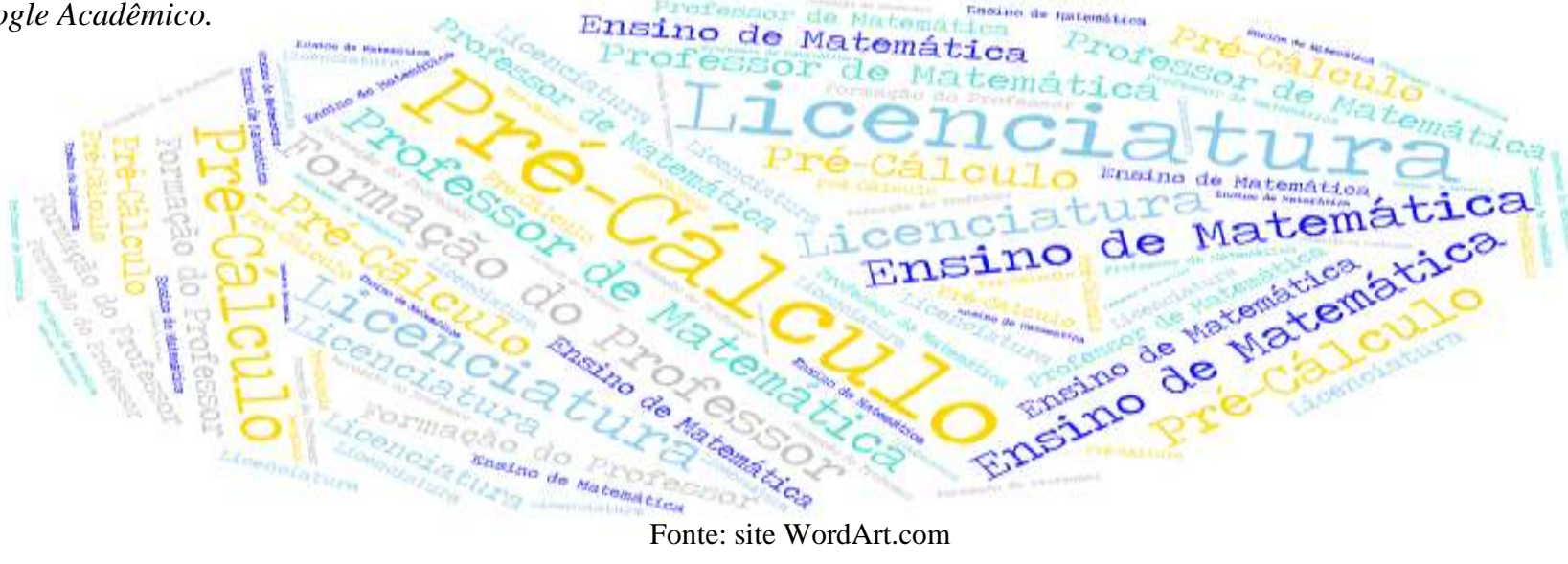

Pode-se observar que os termos encontrados (Figura 2), subsidiaram a construção dos argumentos nesta pesquisa, que acenam para a constatação das publicações que abordam a importância do componente curricular Pré-Cálculo no contexto das ciências exatas. Configurando a presença das instituições de ensino que buscam formas e métodos ao desenvolvimento dos estudantes que recém ingressam na universidade.

No Sistema Educacional do Brasil tem ocorrido constantes mudanças nos currículos dos cursos superiores, bem como nos demais níveis da educação, a exemplo da BNCC que regulamenta possibilidades instrumentais para inserção de novos métodos de ensino com atribuições definidas para cada área do conhecimento. Apesar disto o discurso em meio acadêmico massivamente demonstra o ensino de matemática enfatizando o desenvolvimento e demonstrações de cálculos, aritmética e álgebra são essencialmente exploradas. 
A caracterização de profundas mudanças nos métodos de ensino nos níveis da educação básica, faz-se necessária ao contexto atual, pois romper com o modelo tradicional de ensino é possibilitar avanços na perseguição da diminuição de evasão e reprovação nos componentes curriculares que envolvem cálculos. Como este propósito de mudança, estimula-se o desafio em promover novas práticas de ensino (Silva, de Sousa \& de Medeiros, 2020).

Broetto e Santos-Wagner (2019), chamam atenção para os pressupostos praticados quanto a concepção de que os estudantes universitários já possuem base suficiente para o desenvolvimento de componentes curriculares como Cálculo Diferencial e Integral que em sua essência requer conhecimento dos conceitos de números irracionais, para seguir com novos conceitos acerca de limite, por exemplo. O distanciamento que reside nos métodos e didáticas antecessores ao nível universitário contribui para um cenário de evasão, desistência e descontentamento por parte de todos os atores envolvidos no ensino das ciências exatas (Silva, 2017).

Neste percurso metodológico cabe refletir sobre novas modalidades de ensino que demonstrem a conduta proativa por parte dos órgãos formadores e por parte do público alvo que relacionado direto e indiretamente com o desenvolvimento educacional, social e político. Motivo pelo qual se emerge oportunizar a prática de novos modelos de ensino recorrendo aos meios tecnológicos (Albrecht \& Maciel, 2020).

A formação do professor deve ser parte indissociável desde a educação básica, não obstante, lembrar que o alicerce de uma profissão se dá no início de sua formação pessoal e cidadã (Barbosa \& Barboza, 2019). O aporte da legislação brasileira denota alguns aspectos para aprimoramento da educação no país, mas não direciona nenhuma ação para efetivação de mudanças, na prática.

Neste sentido, Morais e Valente (2020) citam ser parte integrante do ser professor a compreensão da complexidade que envolve as múltiplas dificuldades dos estudantes na realização de exercícios propostos para solução de problemas, alinhado ao contexto social do estudante torna-se possível enveredar por estabelecimento de propostas didáticas que colaborem para desenvolvimento do conhecimento de forma ampla e reflexiva.

Porém, pensar em melhorias para o ensino e aprendizagem dos estudantes universitários no componente curricular Pré-Cálculo sem considerar a inserção do professor às novas modalidades de ensino como o Ensino Híbrido e Ensino Remoto, que exigem conhecimentos de tecnologias educacionais (Silva \& Andrade, 2021), é contribuir para a existência de fenômenos dos tipos, emocionais, psicológicos, desinteresse, evasão, desistência, dentre tantos outros, ou seja, deve-se lembrar que o estudante universitário de hoje é um potencial professor no futuro profissional.

\section{Considerações Finais}

O ensino de matemática caracteriza-se por uma área profissional que se infere em diversas áreas do conhecimento, através de operações matemáticas são apresentados resultados nas mais variadas demonstrações que possibilitam responder aos anseios da sociedade, seja para questões econômicas, de desenvolvimento, ampliações, como soluções exequíveis para problemas complexos.

Esta pesquisa favorece na compreensão sobre o componente curricular Pré-Cálculo, desde sua magnitude e importância no contexto educacional, bem como na contribuição para vida. Foi possível perceber que o desenvolvimento deste componente merece atenção dos professores, estudantes e os demais atores envolvidos na promoção de uma educação eficiente e fundamental ao desenvolvimento de um país. Contudo, o estudo contribui no desenvolvimento conceitual de PC como componente que não se caracteriza um problema situacional, e sim a oportunidade para desenvolvimento inicial na formação de futuros professores.

Com a análise do arcabouço teórico perceberam-se didáticas e metodológicas massivamente tradicionais no modelo de ensino, fato que demonstra a necessidade de mudanças neste cenário. Motivo pelo qual, o ensino deste componente 
curricular enseja reflexões em sua aplicabilidade não somente no âmbito escolar, como fora dele. Tal fato decorre da iminente necessidade de novas perspectivas e necessidades para a observação crítica e intervencionista com políticas públicas que permitam flexibilizar modelos de ensino com base na construção reflexiva e não reprodutiva. Perspectiva que remonta o novo pensar nas questões que envolvem a formação do professor de matemática, desde a concepção e elaboração das ementas de componentes curriculares ao contexto do professor que colocará em prática tais conteúdos do ementário.

Encerra-se este estudo sugerindo o desenvolvimento de novas reflexões sobre o conjunto de dados que contemplaram a base argumentativa e analítica constantes em toda estrutura desta pesquisa. Pesquisas aprofundadas sobre a formação do professor de licenciatura ou bacharelado em matemática e as didáticas praticadas no ensino de Pré-Cálculo, se apresentam imprescindíveis.

\section{Agradecimentos}

Ao Instituto Federal de Educação, Ciência e Tecnologia de Pernambuco - IFPE; ao Programa Universidade Aberta do Brasil - UAB; à Coordenação de Aperfeiçoamento de Pessoal de Nível Superior - CAPES.

\section{Referências}

Albrecht, E., \& Maciel, M. D. (2020). Educação cts e educação matemática crítica nas diretrizes para os cursos de licenciatura em matemática. Research, Society And Development, 9(7), 1-17. Https://Doi.Org/10.33448/Rsd-V9i7.4308

Andrade, F., Esquincalha, A., \& de Oliveira, A. T. (2019). O Pré-Cálculo nas licenciaturas em matemática das instituições públicas do rio de janeiro: o prescrito. VIDYA, 39(1), 131-151. https://doi.org/10.37781/vidya.v39i1.2417

Barbosa, D. E. F., \& Barboza, P. L. (2019). A formação do professor de matemática: buscando caminhos para superar as dificuldades no início da carreira. Research, Society and Development, 8(3), 1-14. https://doi.org/10.33448/rsd-v8i3.794

Bardin, L. (2011). Análise de conteúdo. Edições 70.

Bianchini, B. L., Lima, G. L. D., \& Gomes, E. (2019). Formação de Professor: reflexões da educação matemática no ensino superior. Educação \& Realidade, 44(1). https://doi.org/10.1590/2175-623677732

Biazutti, A., Vaz, R. F. N. \& Andrade, L. R. P. (2020). Discutindo o Método de Ensino por meio da Resolução de Problemas. Revista Baiana de Educação Matemática. 1, 1-23. https://doi.org/10.47207/rbem.v1i.10316

Broetto, G. C., \& Santos-Wagner, V. M. P. D. (2019). O Ensino de Números Irracionais na Educação Básica e na Licenciatura em Matemática: um círculo vicioso está em curso?. Bolema: Boletim de Educação Matemática, 33(64), 728-747. https://doi.org/10.1590/1980-4415v33n64a14

Brasil, Mistério da Educação. Base nacional comum curricular. MEC, Secretaria de Educação Básica, 2017

da Silva Saadi, A., Machado, C. C., \& Pereira, E. C. (2020). Uma prática pedagógica no ensino de funções utilizando o geogebra em um curso de pré-cálculo híbrido. Revista Eletrônica De Educação Matemática, 15(2), 1-18.Https://Doi.Org/10.5007/1981-1322.2020.E70522

de Andrade, F. C., \& da Conceição Esquincalha, A. (2020a). O que dizem os Professores das Licenciaturas em Matemática sobre suas Práticas e Percepções em Pré-Cálculo? What do mathematics degree professors say about their practices and perceptions in Precalculus?. Educação Matemática Pesquisa: Revista do Programa de Estudos Pós-Graduados em Educação Matemática, 22(2), 573-603. https://doi.org/10.23925/1983-3156.2020v22i2p573-603

de Andrade, F. C., \& Da Conceição Esquincalha, A. (2020b). Um Estado Da Arte Das Pesquisas Brasileiras Sobre Pré-Cálculo. Revista Boem, 8(16), 91-111. Https://Doi.Org/10.5965/2357724x08162020091

de Andrade, F. C., \& da Conceição Esquincalha, A. D. C. (2020c). Percepção de estudantes acerca de pré-cálculo na licenciatura em matemática. Revista Paranaense de Educação Matemática, 9(19), 172-197. http://revista.unespar.edu.br/index.php/rpem/article/view/673

de Jesus, R. P. D. S., \& SOUTO, D. L. P. (2018). Pré-cálculo e cálculo diferencial e integral iii com vídeos na educação a distância. Simpósio Internacional de Tecnologias em Educação Matemática (SITEM), 30, 1-49. https://sitem2018.webnode.com/_files/200000110-056390563c/_Anais_do_SITEM.pdf

de Medeiros Rosa, C., Alvarenga, K. B., \& dos Santos, F. F. T. (2019). Desempenho acadêmico em cálculo diferencial e integral. Revista Internacional de Educação Superior, 5, 1-16. https://doi.org/10.20396/riesup.v5i0.8653091

de Moraes, U. C., Azevedo, V. L. A., da Silva Vieira, M. M., \& Abar, C. A. (2019). Projeto pré-cálculo: reforço matemático para os cursos de engenharia em trilhas de aprendizagem do ensino híbrido. Brazilian Applied Science Review, 3(1), 269-281. https://doi.org/10.20396/riesup.v5i0.8653091

de Oliveira, R. A., Gonçalves, W. V., \& Piasson, D. (2018). O uso do Geogebra para o ensino de cálculo diferencial e integral, um mapeamento de suas publicações. Revista Thema, 15(2), 466-484. https://doi.org/10.15536/thema.15.2018.466-484.892 
Diefenthäler, A. T. (2017). Disciplina pré-cálculo: um olhar a partir do desempenho dos acadêmicos. Revista da Universidade Regional do Noroeste do Estado do Rio Grande do Sul, 1-27. http://bibliodigital.unijui.edu.br:8080/xmlui/handle/123456789/4238

dos Santos Rocha, C. E., Santarosa, M. C. P., \& Spohr, C. B. (2018). Projeto Pré-Cálculo na Universidade Federal de Santa Maria: reflexões sobre a contribuição para acadêmicos da matemática e outras áreas científicas. Revista Thema, 15(3), 1154-1163. https://doi.org/10.15536/thema.15.2018.11541163.924

dos Santos, L. M., Henning, E., Taschetto, M. P., \& Girardi, A. (2020). Aspectos do ensino e da aprendizagem de matemática no ensino superior: entre conteúdos, práticas e pesquisas. Revista Boem, 8(16), 1-12. HTTPS://DOI.ORG/10.5965/2357724X08162020001

Esquincalha, A., de Andrade, F. C., \& de Oliveira, A. T. C. C. (2020). Un estado del conocimiento de las investigaciones sobre pre-cálculo en Brasil. UniónRevista Iberoamericana de Educación Matemática, 16(58), 48-63. https://union.fespm.es/index.php/UNION/article/view/99

Gomes, M. L. M. (2018). Elementos de uma História de Formação Docente: as memórias de um professor de Matemática. Bolema: Boletim de Educação Matemática, 32(60), 191-211. https://doi.org/10.1590/1980-4415v32n60a10

Guérios, E., \& Gonçalves, T. O. (2019). Um estudo acerca da pesquisa sobre formação inicial de professores que ensinam matemática nos anos iniciais de escolarização. Educar em Revista, 35(78), 27-45. https://doi.org/10.1590/0104-4060.68973

Lopes, R. P., \& Fürkotter, M. (2020). Do projeto pedagógico à aula universitária: aprender a ensinar com tdic em cursos de licenciatura em matemática. Educação em Revista, 36, 1-31. https://doi.org/10.1590/0102-4698220954

Mazo, A., Oliveira, R. K. D., \& Tomazzoni, E. L. (2021). Análisis bibliográfica y sistemática de la literatura académica de los términos “ciudades inteligentes", "turismo" y "competitividad". Turismo: Visão e Ação, 23(1), 148-168. https://doi.org/10.14210/rtva.v23n1.p148-168

Menezes, A. H. N., Duarte, F. R., Carvalho, L. O. R., \& Souza, T. E. S. (2019). Metodologia científica: teoria e aplicação na educação a distância. Universidade Federal do Vale do São Francisco, Petrolina-PE. e-book, 83p.

Morais, R. D. S., \& Valente, W. R. (2020). Os Experts e o Saber Profissional do Professor que Ensina Matemática. Ciência \& Educação (Bauru), 26, 1-13. https://doi.org/10.1590/1516-731320200029

Pereira, A. S. et al. (2018). Metodologia da pesquisa científica. UFSM. https://repositorio.ufsm.br/bitstream/handle/1/15824/Lic_Computacao_MetodologiaPesquisa-Cientifica. pdf.

Saccaro, A., França, M. T. A., \& Jacinto, P. D. A. (2019). Fatores Associados à Evasão no Ensino Superior Brasileiro: um estudo de análise de sobrevivência para os cursos das áreas de Ciência, Matemática e Computação e de Engenharia, Produção e Construção em instituições públicas e privadas. Estudos Econômicos, 49(2), 337-373. https://doi.org/10.1590/0101-41614925amp

Silva, A. G. S., de Sousa, F. J. F., \& de Medeiros, J. L. (2020). O ensino da matemática: aspectos históricos. Research, Society and Development, 9(8), 1-18. https://doi.org/10.33448/rsd-v9i8.5850

Silva, E. S., \& Andrade, S. D. (2021). A Ótica do Professor Formador sobre a Integração das Tecnologias à Licenciatura em Matemática. Ciência \& Educação (Bauru), 27, 1-11. https://doi.org/10.1590/1516-731320210006

Silva, G. H. G. D. (2017). Educação matemática e ações afirmativas: possibilidades e desafios na docência universitária. Cadernos de Pesquisa, 47(165), 820846. https://doi.org/10.1590/198053143986

Silva, T. M., da Silva Cardoso, E., Marins, G. G., Soares, D. T., Viana, C. A., \& Tolfo, D. D. R. (2019). Relato de experiência: projeto de ensino-relembrando conceitos de matemática básica (RMB). Anais do Salão Internacional de Ensino, Pesquisa e Extensão, 10(1), 1-7. https://guri.unipampa.edu.br/uploads/evt/arq_trabalhos/16601/seer_16601.pdf

Thomé, V. W., Duro, M. L., \& Andrade, C. L. (2020). História da Análise Matemática e Desenvolvimento Cognitivo. Bolema: Boletim de Educação Matemática, 34(67), 399-420. https://doi.org/10.1590/1980-4415v34n67a03

Torres, A. F. N., \& Soares, L. H. (2017). Análise de erros de calouros da licenciatura em matemática em assuntos pré-cálculo. In Vii Congresso Internacional De Ensino De Matemática-2017. Portal de Eventos - ULBRA - CANOAS - Rio Grande do Sul, 1-14. http://www.conferencias.ulbra.br/index.php/ciem/vii/paper/view/7741/0

Vargas, A. P., Santos, R., Neves, M., Teixeira, D., \& da Costa Botelho, S. S. (2020). Sistema de recomendação baseado no ELO para problemas de précálculo: Um experimento com calouros universitários. In Anais do XXXI Simpósio Brasileiro de Informática na Educação (pp. 1213-1222). SBC. https://doi.org/10.5753/cbie.sbie.2020.1213 